Planetary Systems in the Universe - Observation, Formation and Evolution

Proceedings IAU Symposium No. 202, (C)2004 IAU

Alan Penny, Pawel Artymowicz, Anne-Marie Lagrange, \& Sara Russell, eds.

\title{
Indicator of Exo-Solar Planet(s) in the Circumstellar Disk Around $\beta$ Pictoris
}

\author{
Nick Gorkavyi \\ NRC/NAS; Code 685, NASA/GSFC, Greenbelt, MD 20771, USA \\ Sara Heap \\ Code 681, NASA/GSFC, Greenbelt, MD 20771, USA \\ Leonid Ozernoy \\ 5C3, George Mason University, Fairfax, VA 22030-4444, USA \\ Tanya Taidakova \\ Computational Consulting Service, MD 20740, USA \\ John Mather \\ Code 685, NASA/GSFC, Greenbelt, MD 20771, USA
}

\begin{abstract}
Our efficient numerical approach has been applied to modeling the asymmetric circumstellar dust disk around $\beta$ Pictoris as observed with the HST/STIS. We present a new model on the origin of the warping of the $\beta$ Pic disk. We suggest that the observed warp is formed by the gravitational influence of a planet with a mass of about 10 masses of Earth, at a distance of $70 \mathrm{AU}$, and a small inclination $\left(\sim 2.5^{\circ}\right)$ of the planetary orbit to the main dust disk. Results of our modeling are compared with the STIS observations.
\end{abstract}

\section{Introduction}

In circumstellar disks, the major sources of dust are thought to be minor bodies, like comets. Both the stellar radiation drag (Poynting-Robertson drag) and stellar wind drag tend to induce dust inflow toward the star. As the dust passes by planets in its infall, it interacts with them, for example, by accumulating in planetary resonances (Liou \& Zook 1999, Ozernoy et al. 2000). The inner region of the $\beta$ Pictoris dusty disk is tilted by a few degrees with respect to the outer disk (Burrows et al. 1996, Mouillet et al. 1997, Heap et al. 2000) (for references, see the last paper). Heap et al. (2000) concluded that the size and shape of the warp favors the presence of a planet(s) in a slightly inclined orbit. 


\section{Modelling $\beta$ Pictoris Disk with Embedded Planet}

The dynamics of interplanetary particles are determined by several effects, including stellar radiation pressure; the Poynting-Robertson (P-R) and stellar wind drags; resonance effects by planets; and gravitational encounters with planets.

To simulate the gravitational dynamics of comets and nonconservative dynamics of dust particles around a star, we use an implicit second-order integrator (Taidakova \& Gorkavyi, 1999). In the absence of a planet, the surface density of dust between the star and the pericenter of dust sources is constant, while the number density of dust varies as $r^{-1}, r$ being heliocentric distance (Gorkavyi et al. 1997). The presence of a planet induces dramatic changes in the cometary and dust distribution. This is illustrated in Fig. 1, which shows a representative trajectory of dust particles.

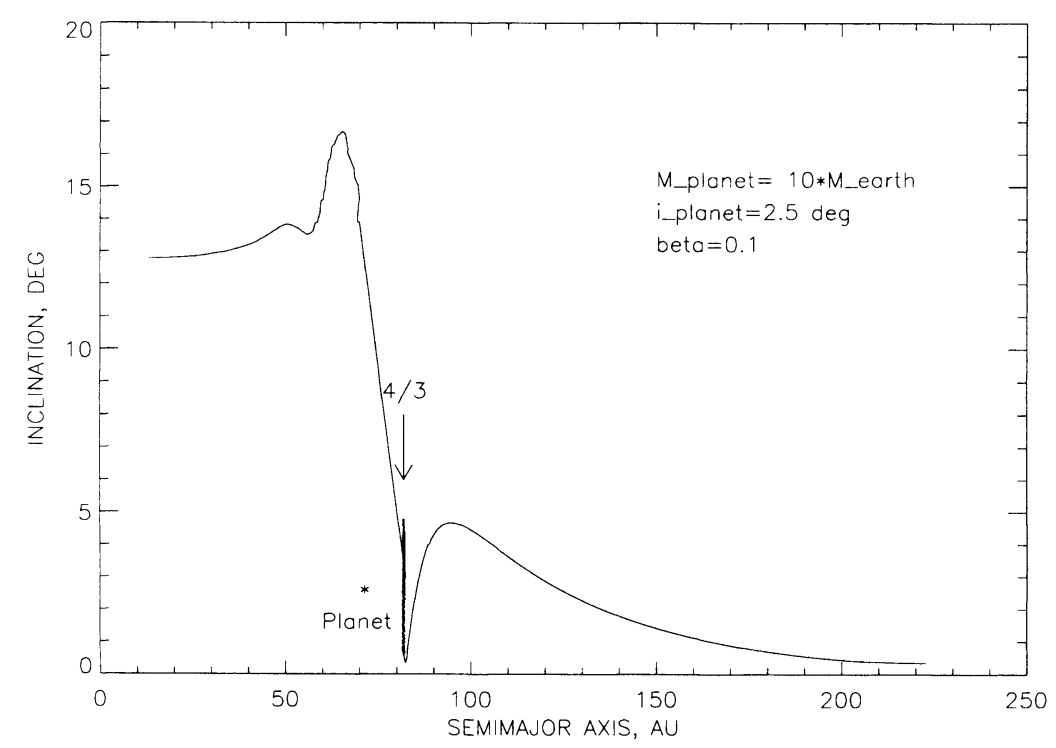

Figure 1. Representative trajectory of a dust particle $(\beta=0.1)$ in the disk of $\beta$ Pic. P-R drag and a slow precession of the particle's orbit around the orbital plane of the planet $\left(a=70 \mathrm{AU} ; i=2.5^{\circ}\right)$ are the dominating dynamical processes during its initial inflow (220-83 AU). The particle's inclination oscillates from 0 to $2 i_{\text {planet }}=5^{\circ}$. The vertical line marks the resonant capture into the $4: 3$ resonance. The next 'jump' to $\sim 17^{\circ}$ is due to scattering of the particle by the planet. The final part of trajectory $(<60 A U)$ marks the particle's drift. After strong interactions with the planet, dust particles (and comets) moves symmetrically about the new plane of the planetary orbit. 


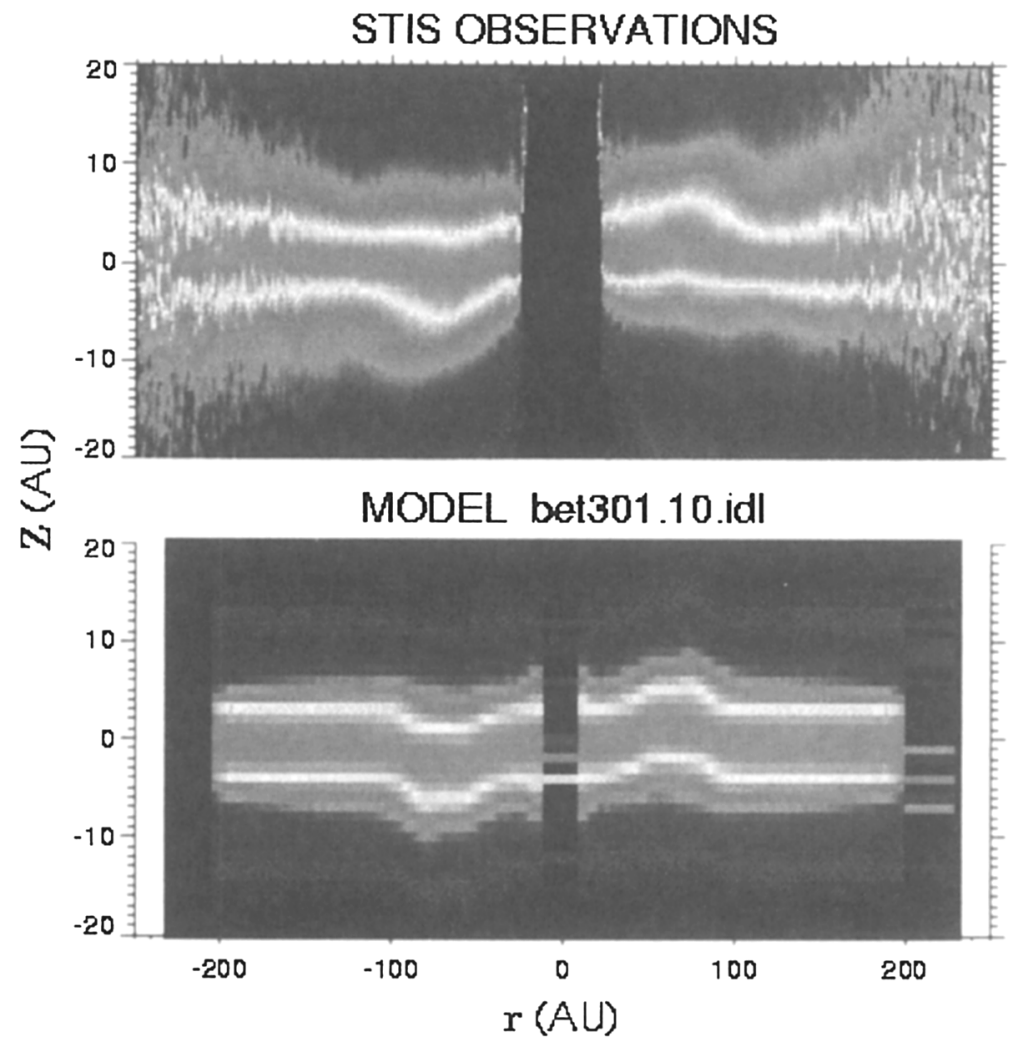

Figure 2. Warp of the inner part of the $\beta$ Pictoris disk. Upper panel: STIS/CCD coronagraphic images of the $\beta$ Pictoris disk normalized to the maximum flux with the vertical scale expanded by $4 \mathrm{X}$ (Heap et al. 2000). Bottom panel: model disk with $M_{p l}=10 M_{\oplus}$, orbital radius $r=70 \mathrm{AU}$, and inclination $i=2.5^{\circ}$. The lifetime of dust particles due to collisions could be as small as 100 years. The stellar radiation pressure is negligible (dust particles are large enough). This warp is stationary, in contrast to the model of Larwood and Papaloizou (1997).

Two algorithms for modeling the $\beta$ Pictoris disk were considered. Algorithm I:

1. Input an initial symmetric cometary disk.

2. Input the mass and orbital radius of the planet on an orbit inclined to the initial cometary disk.

3. Simulate the asymmetric dust distribution on a time scale of $1.4 \times 10^{6}$ yrs. Inclination of particles orbits to the plane of the initial cometary disk and asymmetry of dust is the result of precession of drifting particles around the planet's orbital plane (see Fig. 1).

4. Simulate the dust-scattered light distribution. 
Algorithm II:

1. Input an initial symmetric cometary disk.

2. Input the mass and orbital radius of the planet on an orbit inclined to the initial cometary disk.

3 . Determine the asymmetric distribution of comets due to orbital precession and gravitational scattering by the planet (these processes can be realized without any drifts) after $2.8 \times 10^{6}$ yrs.

4. Simulation of the asymmetric dust distribution for short life times of particles. Asymmetry of dust is mainly a result of an asymmetry in the initial cometary distribution.

5. Simulate the dust-scattered light distribution. (see Fig. 2).

Both alternatives produce a similar warp, but the second alternative seems to be more preferable due to:

- a more realistic (shorter) lifetime of particles;

- accounting for gravitational interactions between comets and the planet.

The STIS images show the disk to be warped at small distances to the star in a sense that the inner disk is tilted by $4.6^{\circ}$ with respect to the outer disk (Heap et al. 2000). See Fig. 2 to compare the STIS observations with our modeling.

\section{Conclusions}

Asymmetric structures in the circumstellar disk indicate at least one planet embedded in the disk of $\beta$ Pictoris.

Acknowledgements. N.N.G. has been supported through NAS/NRC Associateship Research program and the STIS Science Team at Goddard. NASA grant NAG-7065 to George Mason University is gratefully acknowledged.

\section{References}

Gorkavyi, N.N., Ozernoy, L.M., Mather, J.C. \& Taidakova, T.A. 1997, ApJ, 488, 268

Heap, S.R., Lindler, D.J., Lanz, T.M., Cornett, R., Hubeny, I., Maran, S.P. \& Woodgate, B. 2000, ApJ, 539, 435

Liou, J.-C. \& Zook, H.A. 1999, AJ, J. 118, 580

Ozernoy, L.M., Gorkavyi, N.N., Mather, J.C. \& Taidakova, T.A. 2000, ApJ, 537, L147 Larwood J.D. \& Papaloizou, J.C.B. 1997, MNRAS, 285, L 288

Taidakova, T.A. \& Gorkavyi, N.N. 1999, in The Dynamics of Small Bodies in the Solar Systems: A Major Key to Solar Systems Studies, Eds. by B.A. Steves and A.E. Roy, NATO ASI Series, Series C. Vol. 522, Kluwer Academic Publ., p.393 